\title{
Test for Basis-Set Errors in Relativistic Dirac-Fock-Slater Calculations with a Numerical AO-DFS-Basis*
}

\author{
W. -D. Sepp and B. Fricke \\ Physics Department, University of Kassel, Heinrich-Plett-Str. 40, D-3500 Kassel, West Germany
}

Received September 19, 1986; accepted November 14, 1986

\begin{abstract}
A fully relativistic four-component Dirac-Fock-Slater program for diatomics, with numerically given AO's as basis functions is presented. We discuss the problem of the errors due to the finite basis-set, and due to the influence of the negative energy solutions of the Dirac Hamiltonian. The negative continuum contributions are found to be very small.
\end{abstract}

\section{Introduction}

The development of $a b$ initio relativistic molecular codes is necessary in order to understand essential areas of molecular and atomic scattering physics in a more quantitative way. The first area is the influence of relativity on the chemistry of molecules which contain one or more heavy atoms. The second area is the physics of nearly adiabatic ion-atom collisions where - when inner shells are involved - even for small $Z$ nuclei the effect of relativity becomes important. So far various approximations have been used to include relativity in molecular calculations. A list of references is given in Ref. [1].

Because we are mainly interested in the ion-atom scattering problem, we restricted ourselves to the development of a two-dimensional program which allows to calculate diatomic or linear molecules at all interatomic distances.

One of the major problems was the selection of the type of basis functions. The experience of quantum-chemistry in the choice of optimized basis-sets (Slater- or Gauss-type functions) is primarily restricted to distances close to or larger than the equilibrium distance, and to non-relativistic systems. Only recently optimized Gaussian basis-sets for relativistic calculations have been presented $[2,3]$. But for the scattering process we need a solution of the problem for internuclear distances between zero and chemical distances. We, therefore, have chosen numerical atomic DFS-functions, which allow a more physical adaptation to the various distances.

In the first part of the paper we describe the Dirac-FockSlater method as well as the choice of our basis and the pre-orthogonalization procedure. In the main part of this paper we discuss a measure for the errors of the energy eigenvalues due to the incompleteness of the basis-set (truncation errors), and due to the contribution of the negative energy continuum (variational collapse errors).

\section{The Dirac-Fock-Slater method and numerical procedure}

The general method of a relativistic DFS-calculation in molecules has been described in various papers [4-8], for details see Ref. [4]. Therefore, only those formulas needed for the

\footnotetext{
* Paper presented at the Adriatico conference "Relativistic Many-Body Problems", Triests, June 30-July 4, 1986.
}

description of the basis-set errors are given:

The one-particle Dirac-Fock-Slater (DFS) equation reads:

$\hat{h}^{\mathrm{DFS}} \psi_{v}(\boldsymbol{r}, s)=\varepsilon_{v} \psi_{v}(\boldsymbol{r}, s), \quad v \in$ occ.

The effective one-particle DFS-operator is given by

$\hat{h}^{\mathrm{DFS}}=c \boldsymbol{\alpha} \cdot \boldsymbol{p}+\beta m c^{2}+\hat{\boldsymbol{V}}^{\mathrm{nuc}}+\hat{\boldsymbol{V}}^{\mathrm{c}}+\hat{\boldsymbol{V}}^{\mathrm{ex}}$,

with $\boldsymbol{\alpha}$ and $\beta$ the usual Dirac matrices. The operator $\hat{V}^{\text {nuc }}$ represents the electron-nucleus interaction potential

$\hat{V}^{\text {nuc }}=-e^{2} \sum_{K} \frac{Z_{K}}{\left|\boldsymbol{r}-\boldsymbol{R}_{K}\right|}$.

The direct potential part is given by

$\hat{V}^{\mathrm{c}}(\boldsymbol{r})=\int \mathrm{d} \boldsymbol{r}^{\prime} \frac{\varrho\left(\boldsymbol{r}^{\prime}\right)}{\left|\boldsymbol{r}-\boldsymbol{r}^{\prime}\right|}$,

and the exchange potential part by

$\hat{V}^{\mathrm{ex}}(\boldsymbol{r})=-3 \alpha\left(\frac{3}{8 \pi} \varrho(\boldsymbol{r})\right)^{1 / 3}$,

with the electron density

$\varrho(\boldsymbol{r})=e^{2} \sum_{v \in \mathrm{occ}} \psi_{v}^{+}(\boldsymbol{r}, s) \psi_{v}(\boldsymbol{r}, s)$.

The solution of the coupled differential equations (1) has to be found by SCF-iterations. To actually solve the equations for the two-dimensional case we expanded the molecular wave functions in atomic basis functions. The wave functions $\psi_{v}$ are expanded first in symmetry orbitals $\chi_{2}$

$\psi_{v}(\boldsymbol{r}, s)=\sum_{i} \chi_{\hat{i}}(\boldsymbol{r}, s) \cdot c_{i v}$

then the symmetry orbitals are expanded in atomic basis functions $\varphi_{k}$, which are Dirac--spinors

$\chi_{\lambda}(\boldsymbol{r}, a)=\sum_{k} \varphi_{k}(\boldsymbol{r}, s) \cdot d_{k i}$.

If we insert this into eq. (1), the DFS equations reduce to the matrix eigenvalue problem

hc $=$ Sce

With $\mathbf{h}$ the Fock, $\mathbf{S}$ the overlap, and $\mathbf{c}$ the coefficient-matrix. The eigenvalue matrix $\epsilon$ is diagonal.

As already mentioned above, we use numerical atomic DFS wave functions as basis functions. The disadvantage of this is that all matrix elements have to be calculated numerically. The advantage, on the other hand, is that only a small number of basis functions is sufficient, and that the contributions of the negative continuum will probably be small [9]. In addition, since we are using separated atom as well as united 
atom DFS atomic wave functions, the basis-set already includes the exact eigenfunctions of eq. (1) at the internuclear distance zero as well as infinity. Moreover, the kinetic energy matrix elements can be calculated by a simple integration, thus avoiding numerical differentiation $[5,10]$. The numerical procedure to actually calculate the overlap- and Fockmatrix elements is either a transformed Gauss-Laguerre integration in elliptic hyperbolic coordinates or a multicenter integration. The number of points needed in the twodimensional grid is about $30 \times 40$ in order to obtain an accuracy of $10^{-5}-10^{-6}$. The direct part $V^{\mathrm{c}}$ of the electron-electron potential is calculated from the electron density $\varrho$ by numerical solution of the Poisson equation $[4,11]$.

\section{Choice of specific basis functions and pre-orthogonalization}

In non-relativistic quantum calculations the parameters for optimized sets of basis functions were derived in the past. Such a knowledge is not yet generally available for relativistic calculations. This is even more true for our case due to three reasons: First, we do not use Slater or Gauss-type orbitals, second, we need a good basis for all internuclear distances, third, we also describe molecules with very heavy atoms.

As usual, of course, the first step is a minimal basis-set consisting of fully or partially occupied atomic levels of the separated atoms or - in the case of small internuclear distances - the levels of the united system. Large improvements are obtained by adding basis functions which are approximately $20 \%$ larger or smaller in size than the minimal basis functions. The most substantial improvement of the basis-set especially at small and intermediate internuclear distances is the addition of so-called monopole basis functions, which are a solution of the atomic problem in the monopole part of the molecular nuclear potential [4].

Because the non-orthogonal basis functions are of limited accuracy it is important to pre-orthogonalize them and to remove the linear-dependent part of the basis-set. We incorporated various methods, like the canonical or the GramSchmidt orthogonalization procedure [12], where one still has the possibility to define in which order the basis functions shall be orthogonalized.

The reason to struggle very hard for a physically adequate basis is threefold: First, the use of any finite basis leads to a truncation error which one must try to minimize. Second, the loss of numerical accuracy due to non-orthogonality must be kept small. Third, spurious contributions of the positron continuum to the calculated (occupied) electron eigenfunctions of $\hat{h}^{\text {DFS }}$ have to be kept small. The latter error is known as "variational collapse" [13]. Its connection with the BrownRavenhall desease [14], and the reason why our method includes an implicit projection operator, which excludes the negative energy states of the Dirac operator, is given in Ref. [9]. The basic theoretical papers which deal with the projection operators on the negative energy states are given in Refs. [14] and [15]. The results of actual DFS molecular calculations may be found in Refs. [4], [11], and [16].

\section{Error bounds for the eigenvalues and variance of the Hamiltonian}

In order to get information on the three errors discussed
Table I. Energy eigenvalues and variance of inner shell electrons of $\mathrm{Ar}^{+}-\mathrm{Ar}^{+}$at $\mathrm{R}=0.7$ a.u.

\begin{tabular}{lrr}
\hline State & $\varepsilon_{v}[\mathrm{eV}]$ & $\delta \varepsilon_{v}[\mathrm{eV}]$ \\
\hline $1(1 / 2) g$ & 3207.1 & 8.9 \\
$1(1 / 2) u$ & 3206.3 & 11.6 \\
$2(1 / 2) g$ & 553.4 & 125.6 \\
$2(1 / 2) u$ & 372.2 & 41.7 \\
$1(3 / 2) u$ & 370.9 & 38.0 \\
$3(1 / 2) g$ & 326.5 & 20.4 \\
$3(1 / 2) u$ & 325.4 & 61.3 \\
$4(1 / 2) g$ & 276.0 & 15.3 \\
$1(3 / 2) g$ & 274.0 & 9.2 \\
$4(1 / 2) u$ & 132.5 & 49.2 \\
\hline
\end{tabular}

above we calculated the variance $\delta \varepsilon_{v}$ of the effective oneparticle Hamiltonian $\hat{h}=\hat{h}^{\text {DFS }}$ in all states $\psi_{v}$

$\delta \varepsilon_{v}=\left(\left\langle v\left|\hat{h}^{2}\right| v\right\rangle-\langle v|\hat{h}| v\rangle^{2}\right)^{1 / 2}$.

A vanishing $\delta \varepsilon_{v}$ would mean that the state $\psi_{v}$ is an exact eigenstate of $\hat{h}$. For the occupied states of our calculations on the system $\mathrm{Ar}^{+}-\mathrm{Ar}^{+}$for internuclear distances above 0.5 a.u. this variance was always smaller than or in the order of $100 \mathrm{eV}$ with a typical value of $30 \mathrm{eV}$. As an example the results of a calculation at $R=0.7 \mathrm{a} . \mathrm{u}$. for core electrons are given in Table I. To interprete the calculated non-zero value of the variance $\delta \varepsilon_{v}$ let us consider the following [17]: Let $P$. be the projector onto the subspace $\mathscr{H}_{p}$ of the total Hilbert space $\mathscr{H}$, spanned by the finite number of atomic basis functions used. The projector onto the complementary space $\mathscr{H}_{Q}$ is given by $Q=1-P$. In actual calculations one then constructs the eigenvalues and eigenstates of the Hamiltonian

$\hat{h}_{p p}=P \hat{h} P$

in the subspace $\mathscr{H}_{p}$ :

$\hat{h}_{P P} \psi_{v}^{0}=\varepsilon_{v}^{0} \psi_{v}^{0}, \quad \psi_{v}^{0} \in \mathscr{H}_{P}$.

Using the standard Feshbach formalism the eigenvalue equation in the subspace $\mathscr{H}_{p}$ with the exact $\varepsilon_{v}$ is given by

$\left(\hat{h}_{P P}+\hat{h}_{P Q} \frac{Q}{\varepsilon_{v}-h_{Q Q}} \hat{h}_{Q P}\right) \tilde{\psi}_{v}=\varepsilon_{v} \tilde{\psi}_{v}, \quad \tilde{\psi}_{v} \in \mathscr{H}_{P}$.

Using first order perturbation theory the second term of eq. (13) leads to the correction $\Delta \varepsilon_{v}$ of the eigenvalue $\varepsilon_{v}^{0}$.

$\Delta \varepsilon_{v}=\left\langle\psi_{v}^{0}\left|\hat{h}_{P Q} \frac{Q}{\varepsilon_{v}-\hat{h}_{Q Q}} \hat{h}_{Q P}\right| \psi_{v}^{0}\right\rangle$.

Inserting a complete set of eigenstates $\psi_{\mu}^{0}$ of $\hat{h}_{Q Q}$ in $Q$-space

$\hat{h}_{Q Q} \psi_{\mu}^{0}=\varepsilon_{\mu}^{0} \psi_{\mu}^{0}, \quad \psi_{\mu}^{0} \in \mathscr{H}_{Q}$

into eq. (14) we get

$\Delta \varepsilon_{v}=\sum_{\mu \in Q} \frac{\left\langle\psi_{v}^{0}|\hat{h}| \psi_{\mu}^{0}\right\rangle\left\langle\psi_{\mu}^{0}|\hat{h}| \psi_{v}^{0}\right\rangle}{\varepsilon_{v}-\varepsilon_{\mu}^{0}}$.

We now decompose the total correction (16) into the contributions from states $\psi_{\mu+}^{0}$ with $\varepsilon_{\mu+}^{0}>\varepsilon_{v}$ and from states $\psi_{\mu-}^{0}$ with $\varepsilon_{\mu-}^{0}<\varepsilon_{v}$ :

$\Delta \varepsilon_{v}=\Delta \varepsilon_{v}^{+}+\Delta \varepsilon_{v}^{-}$

with

$\Delta \varepsilon_{v}^{ \pm}=\sum_{\mu \in Q \pm} \frac{\left\langle\psi_{v}^{0}|\hat{h}| \psi_{\mu \pm}^{0}\right\rangle\left\langle\psi_{\mu \pm}^{0}|\hat{h}| \psi_{v}^{0}\right\rangle}{\varepsilon_{\nu}-\varepsilon_{\mu \pm}^{0}}$. 
These partial errors are upper and lower bounds for the error of the energy eigenvalues

$\Delta \varepsilon_{v}^{+} \leqslant \Delta \varepsilon_{v} \leqslant \Delta \varepsilon_{v}^{-}$

since the numerators in eq. (18) are positive definite. We now introduce the mean excitation energies $\varepsilon_{Q_{ \pm}}^{0}$ of the $Q_{ \pm \text {-spaces }}$ which changes eq. (18) to

$\Delta \varepsilon_{v}^{ \pm}=\frac{1}{\varepsilon_{v}-\varepsilon_{Q \pm}^{0}} \sum_{\mu \in Q \pm}\left|\left\langle\psi_{v}^{0}|\hat{h}| \psi_{\mu \pm}^{0}\right\rangle\right|^{2}$.

Extending the summation to the whole $Q$-space yields bounds for the corrections $\Delta \varepsilon_{v}^{ \pm}$

$\Delta \varepsilon_{v}^{ \pm}\left(\varepsilon_{v}-\varepsilon_{Q \pm}^{0}\right) \leqslant \sum_{\mu \in Q}\left|\left\langle\psi_{v}|\hat{h}| \psi_{\mu}^{0}\right\rangle\right|^{2}$.

Now the summation can be further extended to all states of Hilbert space because

$\left\langle\psi_{v}^{0}|\hat{h}| \psi_{v^{\prime}}^{0}\right\rangle=0 \quad$ for $v \neq v^{\prime} ; \quad v, v^{\prime} \in P$

and we get

$\sum_{\mu \in Q}\left|\left\langle\psi_{\nu}^{0}|\hat{h}| \psi_{\mu}^{0}\right\rangle\right|^{2}=\sum_{\mu}\left|\left\langle\psi_{\nu}^{0}|\hat{h}| \psi_{\mu}^{0}\right\rangle\right|^{2}-\left|\left\langle\psi_{v}^{0}|\hat{h}| \psi_{v}^{0}\right\rangle\right|^{2}=\left(\delta \varepsilon_{\nu}\right)^{2}$.

Equation (21) then reads

$\Delta \varepsilon_{v}^{ \pm}\left(\varepsilon_{v}-\varepsilon_{Q \pm}^{0}\right) \leqslant\left(\delta \varepsilon_{v}\right)^{2}$.

Of course, the mean excitation energies $\varepsilon_{Q_{ \pm}}^{0}$ are not known, but we can get even larger bounds for $\Delta \varepsilon_{v}^{ \pm}$if we replace the differences $\left|\varepsilon_{v}-\varepsilon_{Q \pm}^{0}\right|$ by

$\left|\varepsilon_{v}-\bar{\varepsilon}_{Q \pm}^{0}\right| \equiv \operatorname{Min}_{\mu \in Q \pm}\left|\varepsilon_{v}-\varepsilon_{\mu \pm}^{0}\right| \leqslant\left|\varepsilon_{v}-\varepsilon_{Q \pm}^{0}\right|$.

So we have finally

$\Delta \varepsilon_{v}^{ \pm} \approx \frac{\left(\delta \varepsilon_{v}\right)^{2}}{\varepsilon_{v}-\bar{\varepsilon}_{Q \pm}^{0}}$.

The energy difference $\varepsilon_{\mathrm{v}}-\bar{\varepsilon}_{Q-}^{0}$ for excitations to the negative continuum is (at least) of the order of $2 m c^{2}$. With the values of $30-100 \mathrm{eV}$ for $\delta \varepsilon_{v}$ in the example $\mathrm{Ar}^{+}-\mathrm{Ar}^{+}$as discussed at the beginning of the chapter we find that the error $\Delta \varepsilon_{v}^{-}$is less than $10 \mathrm{meV}$ even if the whole variance $\delta \varepsilon_{v}$ should turn out to be due to contributions of the negative energy part of the basis-set; however, we expect that the main part of $\delta \varepsilon_{v}$ is due to the imcompleteness of the basis-set in the positive energy part. An indication of this supposition is the fact that in all tested cases an increase of the basis set leads to a small decrease of the energy eigenvalues.

An estimate for the error $\Delta \varepsilon_{v}^{+}$is more difficult as there is no gap between the calculated eigenvalues $\varepsilon_{v}^{0}$ and the eigenvalues $\varepsilon_{\mu+}^{0}$ in $\mathscr{H}_{Q_{+}}$. The lower bound of $\varepsilon_{\mu+}^{0}$ in general will be even lower than the upper bound of the calculated eigenvalues $\varepsilon_{v}^{0}$. However, all eigenvalues $\varepsilon_{\mu+}^{0}$ must be higher than those calculated eigenvalues $\varepsilon_{v}^{0}$ which are sufficiently close to the exact eigenvalues. At least this is assured for all the occupied levels as indicated by their relatively low values of the variance $\delta \varepsilon_{v}$. A crude measure for $\bar{\varepsilon}_{Q+}^{0}$ is, therefore, the energy of the last occupied level. Using this bound for the mean excitation energy $\vec{\varepsilon}_{0+}^{0}$ we find that the error, due to the truncation of the basis-set, for instance for K-shell electrons in the scattering system $\mathrm{Ar}^{+}-\mathrm{Ar}^{+}$, is less than $1 \mathrm{eV}$. Of course, no useful bounds for the truncation error for the valence electrons can be given [18] since the energy denominator in eq. (26) is too small.
One problem remains: The errors due to the numerical calculations of the matrix representations of the Hamiltonian $\hat{h}$ are not explicitly discussed in this chapter. Nevertheless, they contribute to the calculatd value of $\delta \varepsilon_{v}$ since eqs. (10) and (23) are not equivalent. This non-equivalence arises because we calculate the matrix elements of $\hat{h}$ and of $\hat{h}^{2}$ independently. So except for accidental cancellation of errors the calculated value of the variance is also a measure for the numerical integration errors.

\section{Conclusions}

The DFS program for diatomic molecules presented here is able to perform quite accurate fully relativistic $a b$ initio calculations in reasonable time. Without using any projection operators onto the positive energy states we are able to demonstrate that:

(a) the basis is sufficiently complete to calculate the inner shell levels with an accuracy of about $\mathrm{leV}$. and

(b) the basis does not contain any observable contributions of the negative energy eigenspace of $\hat{h}$.

This gives us the confidence that this type of calculation is a tool to perform accurate fully relativistic ab initio calculations at the Hartree-Fock-Slater level in molecules or in quasi-molecules, where the inner levels are affected by relativistic effects even for atoms with small $Z$.

\section{Acknowledgements}

We would like to thank Prof. W. H. E. Schwarz for a number of very helpful discussions in the course of this work.

\section{References}

1. Pyykkö, P., Relativistic Theory of Atoms and Molecules - A Bibliography 1916-1985 Lecture Notes in Chemistry, Vol. 41, Springer Verlag 1986

2. Mark, F., Theor. Chim. Acta 70, 165 (1986); Mark, F., Relativistic Gaussian Basis Sets for Atomic-Systems with One to Ten Electrons, Schriftenreihe des Max-Planck-Instituts für Strahlenchemie Nr. 24, Mühlheim-Ruhr (1985).

3. Aerts, P. J. C., Towards Relativistic Quantum Chemistry, Ph.D. Thesis, Groningen (1986).

4. Sepp, W. -D., Kolb, D., Sengler, W., Hartung, H. and Fricke, B., Phys. Rev. A33, 3679 (1986).

5. Averill, F. W. and Ellis, D. E., J. Chem. Phys. 59, 6412 (1973).

6. Rosén, A. and Ellis, D. E., J. Chem. Phys. 62, 3039 (1975).

7. Rosén, A., Ellis, D. E., Adachi, H. and Averill, F. W., J. Chem. Phys. 65, 3629 (1976).

8. Delley, B. and Ellis, D. E., J. Chem. Phys. 76 I949 (1982).

9. Sepp, W. -D. and Fricke, B., Atomic Theory Workshop on Relativistic and QED Effects in Heavy Atoms (Edited by H. P. Kelly and Y. K. Kim), p. 20, Gaithersburg 1985, AIP Conference proceedings No. 136.

10. Ellis, D. E. and Rosén, A., Z. Physik A283, 3 (1977).

11. Sepp, W. -D., Sengler, W., Kolb, D., Hartung, H. and Fricke, B., Chem. Phys. Lett. 109, 233 (1984).

12. Löwdin, P. O., Adv. Phys. 5, 1 (1958).

13. Kutzelnigg, W., Int. J. Quant. Chem. 25, 107 (1984).

14. Brown, G. E. and Ravenhall, D. G., Proc. Roy. Soc. London Ser. A208, 552 (1951).

15. Sucher, J., Phys. Rev, A22, 348 (1980); Int. J. Quantum Chem. 25, 3 (1984).

16. Hartung, H., Fricke, B., Sepp, W. -D., Sengler, W. and Kolb, D., J. Phys. B18, L433 (1985).

17. Weinstein, D. H., Proc. Nat. Acad. Sci. 20, 529 (1934).

18. Wilson, S., Methods in Computational Molecular Physics (Edited by G. H. F. Diercksen and S. Wilson), p. 71, D. Reidel Publishing Company, Dordrecht, 1983, NATO ASI Series C, No. 113. 